\title{
【 LETTERS TO THE EDITOR 】
}

\section{Reply to Comments on Relationship between Hypoalbuminemia on Admission and Long-term Mortality in Patients with Acute Decompensated Heart Failure}

Key words: hypoalbuminemia, acute decompensated heart failure, long-term mortality

(Intern Med 58: 3491, 2019)

(DOI: 10.2169/internalmedicine.3411-19)

The Authors Reply We appreciate the interest of Dr. Imamura in our recent paper, as well as his valuable comments (1). In our study, hypoalbuminemia was defined as a serum albumin level of $\leq 3.4 \mathrm{~g} / \mathrm{dL}$ because the median serum albumin level was $3.4 \mathrm{~g} / \mathrm{dL}$, which is generally accepted as the cutoff value (2-4). In response to Dr. Imamura's initial comment, we performed a receiver-operating characteristic (ROC) curve analysis, and $3.8 \mathrm{~g} / \mathrm{dL}$ was found to be the ideal cutoff value for predicting the outcome in our dataset. However, hypoalbuminemia at admission, defined as a serum albumin level of $\leq 3.8 \mathrm{~g} / \mathrm{dL}$, was not associated with the long-term outcomes [hazard ratio (HR), 1.21; 95\% confidence interval $(\mathrm{CI}), 0.70-2.22 ; \mathrm{p}=0.511$ in the pre-match model, and HR, 0.97; 95\% CI, 0.45-2.07; $\mathrm{p}=0.934$ in the post-match model].

The duration of follow-up varied across individual cases on the basis of the relatively long enrollment period (i.e., 5 years) and thus the numbers at risk decreased with the passage of time. In the Kaplan-Meier survival analyses, the two curves crossed after several years, as Dr. Imamura pointed out. However, the proportional hazards assumption was confirmed in both the pre-match and post-match models using $\log$-minus-log survival graphs. It is often difficult to show the relationship between parameters/conditions in the acute phase and the long-term outcomes. However, many reports have investigated the relationships between parameters/conditions in the acute phase and long-term outcomes $(5,6)$. Again, we thank Dr. Imamura for his interest and comments on our paper.

Author's disclosure of potential Conflicts of Interest (COI). Hiroyuki Daida: Fees for promotional materials, Kirin, Kaken
Pharmaceutical, Abbott Japan, Astellas Pharma, Astra Zeneca, Bayer Yakuhin, Boston Scientific Japan, Bristol-Myers Squibb, Daiichi Sankyo, MSD, Pfizer, Philips Respironics, Sanofi and Takeda Pharmaceutical; Research funding, Kirin, Kaken Pharmaceutical, Abbott Japan, Astellas Pharma, AstraZeneca, Bayer Yakuhin, Boston Scientific Japan, Bristol-Myers Squibb, Daiichi Sankyo, MSD, Pfizer, Philips Respironics, Sanofi and Takeda Pharmaceutical.

\section{Financial Support}

This study was partly supported by a Grant-in-Aid for Scientific Research (C) [Grant Number 26507010] and a grant to the Respiratory Failure Research Group from the Ministry of Health, Labor and Welfare, Japan. These funding sources had no other roles in this study.

\section{Shoichiro Yatsu ${ }^{1}$, Takatoshi Kasai ${ }^{1-3}$ and Hiroyuki Daida ${ }^{1}$}

\section{References}

1. Yatsu S, Kasai T, Matsumoto H, Shitara J, Shimizu M, Murata A. Relationship between hypoalbuminemia on admission and longterm mortality in patients with acute decompensated heart failure. Intern Med 58: 1695-1702, 2019.

2. Uthamalingam S, Kandala J, Daley M, Patvardhan E, Capodilupo R, Moore SA. Serum albumin and mortality in acutely decompensated heart failure. Am Heart J 160: 1149-1155, 2010.

3. Bonilla-Palomas JL, Gamez-Lopez AL, Moreno-Conde M, Lopez-Ibanez MC, Anguita-Sanchez M, Gallego de la Sacristana A. Hypoalbuminemia in acute heart failure patients: causes and its impact on hospital and long-term mortality. J Card Fail 20: 350358, 2014.

4. Ancion A, Allepaerts S, Oury C, Gori AS, Pierard LA, Lancellotti P. Serum albumin level and hospital mortality in acute nonischemic heart failure. ESC Heart Fail 4: 138-145, 2017.

5. Barsheshet A, Shotan A, Cohen E, Garty M, Goldenberg I, Sandach A. Predictors of long-term (4-year) mortality in elderly and young patients with acute heart failure. Eur J Heart Fail 12: 833-840, 2010.

6. Lu DY, Cheng HM, Cheng YL, Hsu PF, Huang WM, Guo CY. Hyponatremia and worsening sodium levels are associated with long-term outcome in patients hospitalized for acute heart failure. J Am Heart Assoc 5: e002668, 2016.

The Internal Medicine is an Open Access journal distributed under the Creative Commons Attribution-NonCommercial-NoDerivatives 4.0 International License. To view the details of this license, please visit (https://creativecommons.org/licenses/ by-nc-nd/4.0/).

\footnotetext{
${ }^{1}$ Department of Cardiovascular Medicine, Juntendo University School of Medicine, Japan, ${ }^{2}$ Cardiovascular Respiratory Sleep Medicine, Juntendo University Graduate School of Medicine, Japan and ${ }^{3}$ Sleep and Sleep-Disordered Breathing Center, Juntendo University Hospital, Japan Received: May 30, 2019; Accepted: June 3, 2019; Advance Publication by J-STAGE: July 22, 2019 Correspondence to Dr. Takatoshi Kasai, kasai-t@mx6.nisiq.net

(C) 2019 The Japanese Society of Internal Medicine. Intern Med 58: 3491, 2019
} 